\title{
TRATAMENTO DE ÁGUAS RESIDUAIS COM CORANTE DISPERSO DA INDÚSTRIA TÊXTIL UTILIZANDO DESTILAÇÃO POR MEMBRANAS
}

\author{
V. H. CORREA ${ }^{1}$, H. RAMLOW ${ }^{2}$, R. C. S. C. VALLE ${ }^{3}$, J. A. B. VALLE ${ }^{4}$, A. C. K. \\ BIERHALZ ${ }^{5}$ e C. MARANGONI ${ }^{6}$ \\ 1,3-6 Universidade Federal de Santa Catarina, Departamento de Engenharia Têxtil \\ 2,4,6 Universidade Federal de Santa Catarina, Programa de Pós-Graduação em Engenharia \\ Química \\ E-mail para contato: v.h.m.c@hotmail.com; heloramlow@gmail.com; rita.valle@ufsc.br; \\ alexandre.valle@ufsc.br; andrea.krause@ufsc.br; cintia.marangoni@ufsc.br.
}

\begin{abstract}
RESUMO - Estudos contemplando o processo de Destilação por Membranas (DM) têm-se desenvolvido nos últimos anos devido à sua utilização eficiente na recuperação de água com possibilidade de reuso. Neste trabalho, investigou-se a aplicabilidade do processo de DM no tratamento de efluente têxtil contendo corantes dispersos. Duas soluções de corante, nas cores preto e vermelho, com $30 \mathrm{mg} \cdot \mathrm{L}^{-1}$ cada foram utilizadas como padrão para representar o efluente. A membrana utilizada neste trabalho foi uma comercial plana de poliamida. Os resultados mostraram que os valores médios de fluxo permeado e de recuperação de corante foram respectivamente $29,3 \mathrm{~kg} \cdot\left(\mathrm{m}^{-2} \cdot \mathrm{h}^{-1}\right)$ e $5,91 \%$ para o corante preto disperso e de $14,16 \mathrm{~kg} \cdot\left(\mathrm{m}^{-2} \cdot \mathrm{h}^{-1}\right)$ e $7,10 \%$ para o corante vermelho disperso, demonstrando a possibilidade de aplicação deste tratamento em águas residuais da indústria têxtil.
\end{abstract}

\section{INTRODUÇÃO}

Um dos maiores desafios mundiais atuais consiste na escassez de água. Este aspecto é extremamente relevante no setor têxtil, devido à grande quantidade necessária de água para atender a demanda de produção e ao aumento da rigorosidade das leis ambientais. Desse modo, tem-se buscado cada vez mais fontes renováveis deste insumo para que a produção industrial caminhe em paralelo com a sustentabilidade ambiental, tornando-se então a busca por alternativas de reuso de água uma área de estudo para os pesquisadores.

A destilação por membranas (DM) é um processo de separação conduzido termicamente no qual apenas moléculas de vapor transferem-se através de uma membrana hidrofóbica porosa do lado de alimentação/retentado e são coletadas, após condensação, no lado permeado/destilado (Alkhudhiri et al., 2011). Isto é, uma diferença de temperatura através das superfícies da membrana induz a diferença de pressão de vapor em ambos os lados. Durante o processo de separação, a fase vapor é mantida dentro dos poros da membrana (Mozia et al., 2009). A natureza da força motriz e a característica hidro-repelente da membrana rejeitam teoricamente $100 \%$ dos solutos não voláteis, como macromoléculas, espécies coloidais, íons inorgânicos e outros compostos não voláteis (Drioli et al., 2014). 
O método de separação de corantes da água através de uma membrana vem se tornando alvo de estudos e investigação voltada para a área têxtil, já que é possível tratar o efluente e reutilizar a água proveniente do sistema de tratamento. No entanto, poucos estudos abordam tal aplicação sendo ainda incipientes os resultados obtidos. (Mokhtar et al., 2016). Dessa forma, o objetivo deste trabalho foi avaliar a recuperação de águas residuais com corantes dispersos aplicando-se o processo de separação de destilação por membranas.

\section{METODOLOGIA}

Os experimentos foram conduzidos em uma unidade experimental em escala laboratorial. $\mathrm{O}$ aparato consiste de um módulo de aço inoxidável contendo uma membrana plana de nanofiltração composta por poliamida (NF90, DOW FILMTEC ${ }^{\mathrm{TM}}$ Membranes). A membrana possui um diâmetro médio de poro de $0,68 \mathrm{~nm}$ (Sert et al., 2016). A área efetiva da membrana é de $0,0177 \mathrm{~m}^{2}$.

Na Figura 1 é apresentado um diagrama esquemático do experimento realizado em laboratório. O sistema foi projetado para ter duas correntes de circulação, isto é, a corrente quente (fluxo de alimentação) e a corrente fria (fluxo de permeado). As temperaturas das soluções foram controladas utilizando-se um aquecedor e um resfriador e foram mantidas aproximadamente a $60{ }^{\circ} \mathrm{C}$ e $20^{\circ} \mathrm{C}$ respectivamente para a alimentação e para o permeado. Soluções de corante preto disperso $\left(30 \mathrm{mg} \cdot \mathrm{L}^{-1}\right)$ e de corante vermelho disperso $\left(30 \mathrm{mg} \cdot \mathrm{L}^{-1}\right)$ foram utilizadas durante os experimentos como solução modelo do efluente têxtil. As vazões médias de alimentação e permeado foram respectivamente $1,65 \mathrm{~L} \cdot \mathrm{min}^{-1}$ e $0,52 \mathrm{~L} \cdot \mathrm{min}^{-1}$. O tempo total do experimento foi de 2 horas e a cada 30 minutos foram retiradas amostras dos tanques de alimentação e permeado para análise. Os experimentos foram realizados em duplicata e os seus valores médios foram utilizados para interpretação dos resultados.

Figura 1 - Diagrama esquemático do experimento de DM

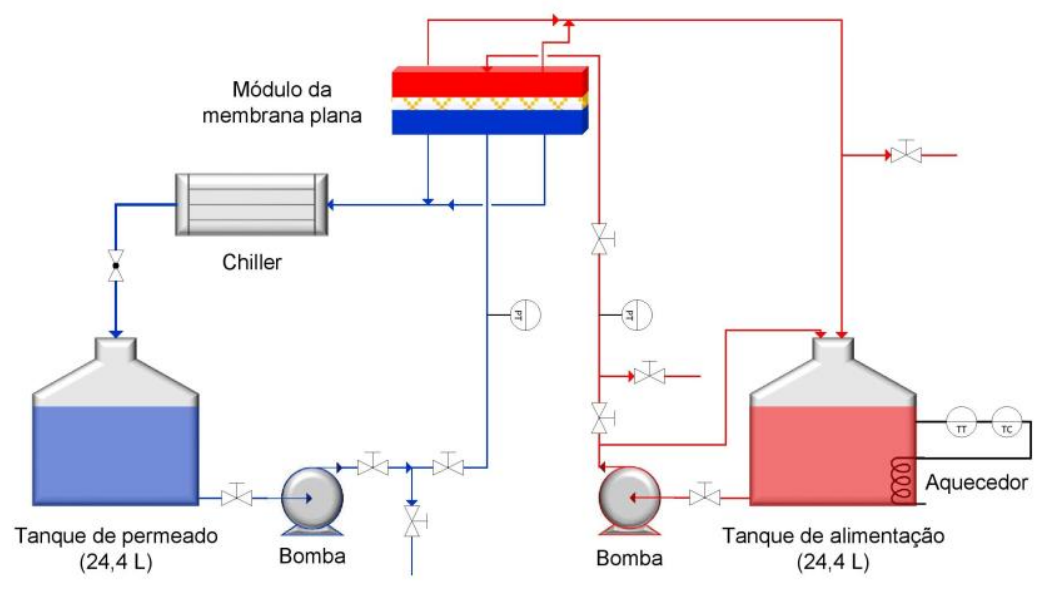

O fluxo permeado (J) foi calculado utilizando a Equação 1, onde $\Delta \mathrm{M}(\mathrm{kg})$ é a massa de permeado coletada em um tempo pré-determinado $\Delta \mathrm{t}(\mathrm{h})$ do processo e $\mathrm{A}\left(\mathrm{m}^{2}\right)$ é a área efetiva da membrana. Avaliou-se o fluxo permeado a cada 30 minutos e o fluxo permeado acumulado de cada medição em relação ao valor no tempo zero. 


$$
J=\frac{\Delta M}{A \times \Delta t}
$$

A recuperação de corante, $\mathrm{R}(\%)$, da membrana foi calculada conforme Equação 2, onde $\mathrm{C}$ é a concentração de corante na alimentação. Avaliou-se a recuperação de corante a cada 30 minutos e a recuperação acumulada de cada medição em relação ao valor no tempo zero.

$$
R(\%)=\left(\frac{C_{F I N A L}-C_{\text {INICIAL }}}{C_{\text {INICIAL }}}\right) \times 100
$$

As concentrações de corante nas amostras de alimentação e de permeado foram obtidas através de uma curva padrão de absorbância versus concentração utilizando um espectrofotômetro UV-vis (AJX 1900, Micronal) medindo a quantidade de luz absorvida em comprimento máximo de onda $(301 \mathrm{~nm})$.

\section{RESULTADOS E DISCUSSÃO}

\subsection{Avaliação do Fluxo Permeado}

Na Figura 2 e na Figura 3 são apresentados os fluxos permeados em função do tempo de separação respectivamente para os corantes preto disperso e vermelho disperso. Os resultados mostraram que há uma recuperação de água no começo do experimento, com um aumento do fluxo de permeado. Porém, após aproximadamente 60 minutos, esse fluxo diminuiu. O fluxo permeado acumulado máximo para o corante preto disperso foi de $38,4 \mathrm{~kg} \cdot\left(\mathrm{m}^{-2} \cdot \mathrm{h}^{-1}\right)$ e para o corante vermelho disperso foi de $18,8 \mathrm{~kg} \cdot\left(\mathrm{m}^{-2} \cdot \mathrm{h}^{-1}\right)$.

Figura 2 - Variação do fluxo permeado acumulado e do fluxo permeado a cada 30 minutos em função do tempo (corante preto disperso)

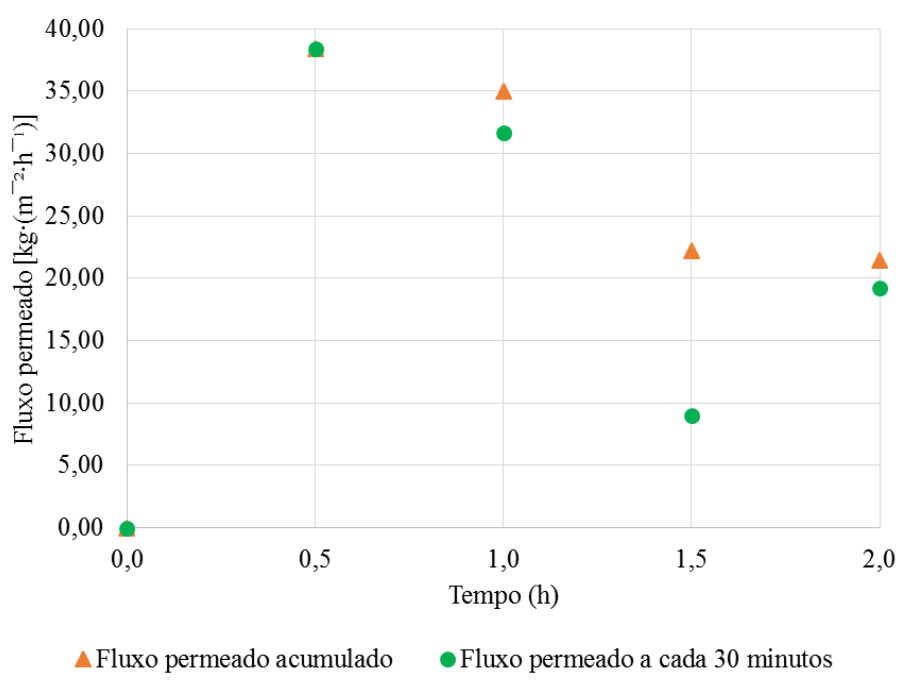


Figura 3 - Variação do fluxo permeado acumulado e do fluxo permeado a cada 30 minutos em função do tempo (corante vermelho disperso)

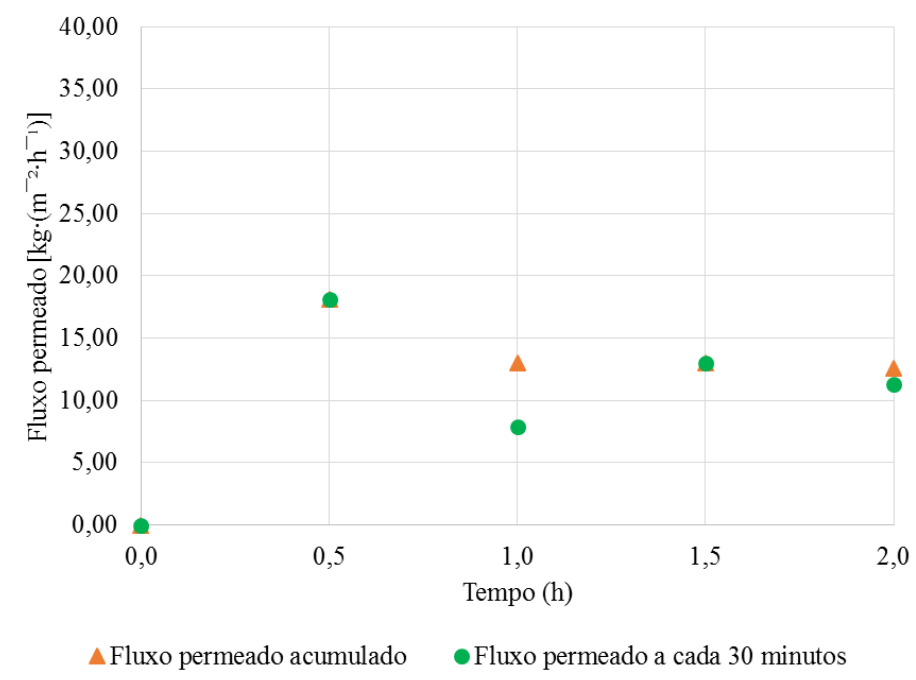

\subsection{Avaliação da remoção de corante}

Na Figura 4 e na Figura 5 são apresentadas as remoções de corante em função do tempo de separação respectivamente para os corantes preto disperso e vermelho disperso. Os resultados mostraram que há uma recuperação acumulada eficiente durante todo o experimento para ambos os corantes.

Figura 4 - Variação da remoção acumulada e da remoção a cada 30 minutos de corante em função tempo (corante preto disperso)

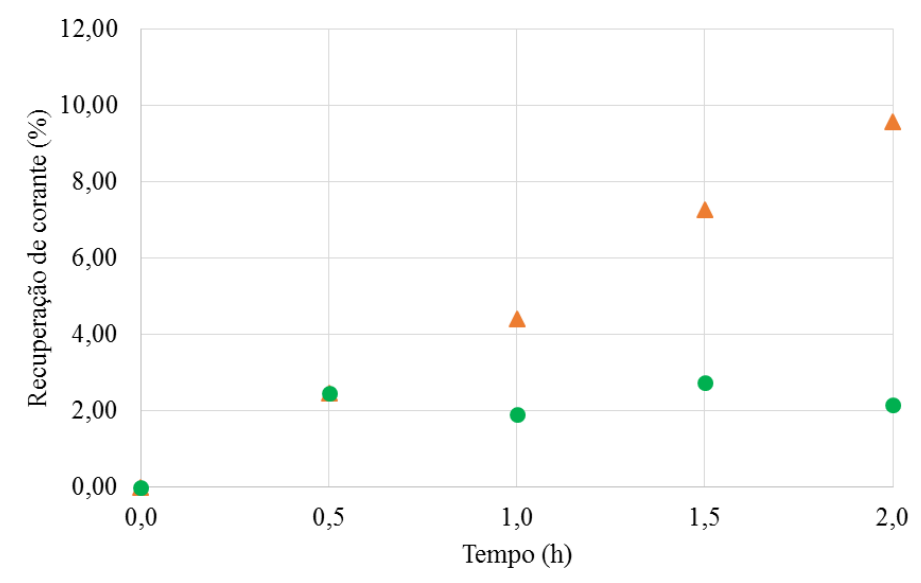

$\triangle$ Recuperação acumulada de corante • Recuperação de corante a cada 30 minutos 


\section{Congresso Brasileiro de Engenharia Química em Iniciação Científica UFSCar - São Carlos - SP 16 a 19 de Julho de 2017}

Figura 5 - Variação da remoção acumulada e da remoção a cada 30 minutos de corante em função do tempo (corante vermelho disperso)

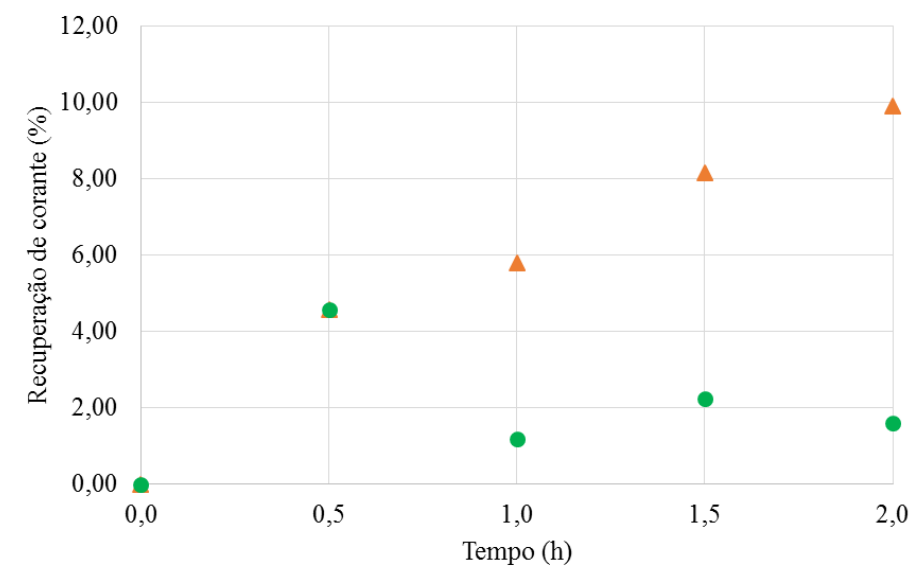

$\Delta$ Recuperação acumulada de corante $\bullet$ Recuperação de corante a cada 30 minutos

\subsection{Comparação entre os corantes}

Na Figura 6 é apresentado um gráfico comparativo do fluxo permeado acumulado e da recuperação acumulada de corante em função do tempo de separação para os corantes preto disperso e vermelho disperso, que pertencem à mesma classe de corantes. Os resultados mostraram que há uma maior recuperação de água no lado permeado para o corante preto disperso. Porém, ao final do experimento, observou-se um declínio do fluxo permeado acumulado para ambos os corantes. Em relação à recuperação acumulada de corante, observou-se um aumento ao longo do tempo para ambos os corantes.

Figura 6 - Comparação da variação do fluxo permeado acumulado e da recuperação acumulada de corante em função do tempo para os corantes preto disperso e vermelho disperso

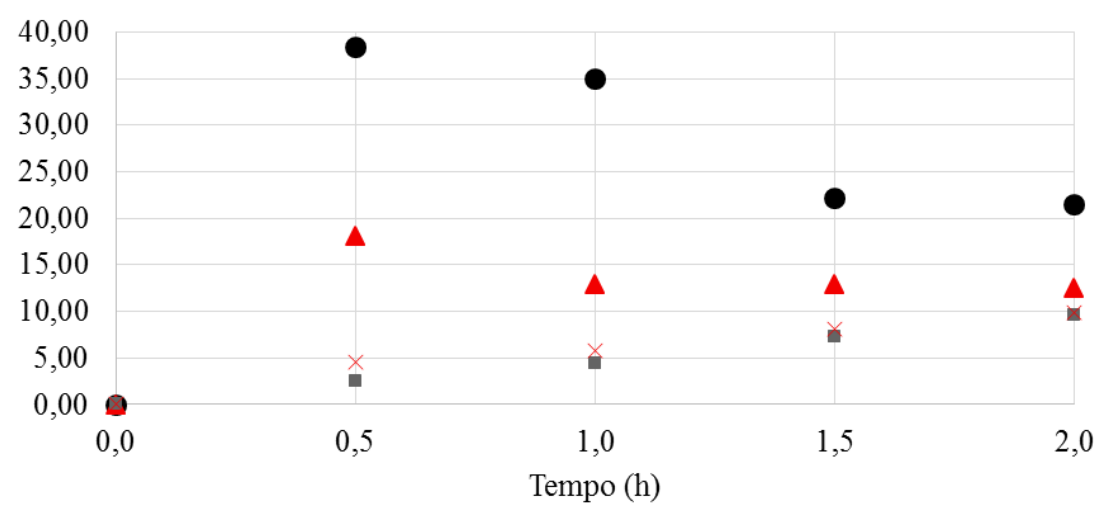

- Fluxo permeado acumulado - preto disperso $\left[\mathrm{kg} \cdot\left(\mathrm{m}^{-2} \cdot \mathrm{h}^{-1}\right)\right]$

$\Delta$ Fluxo permeado acumulado - vermelho disperso $\left[\mathrm{kg} \cdot\left(\mathrm{m}^{-2} \cdot \mathrm{h}^{-1}\right)\right]$

- Recuperação acumulada de corante - preto disperso (\%)

$\times$ Recuperação acumulada de corante - vermelho disperso (\%) 


\section{CONCLUSÃO}

Os resultados dos experimentos mostraram a possibilidade do uso do processo de destilação por membranas para recuperação de água sem a presença de corante no lado permeado. Apesar disso, um rápido declínio do fluxo permeado foi observado, indicando a necessidade de avaliar mais detalhadamente o comportamento do processo.

As condições experimentais empregadas devem ser otimizadas e testadas em uma faixa mais ampla de forma a melhorar a recuperação de água pelo processo. Dessa forma, mais testes precisam ser realizados para melhor entendimento do processo de DM e sua aplicabilidade no tratamento de águas residuais têxteis, já que os dados aqui apresentados são resultados preliminares.

No entanto, ressalta-se a potencialidade de aplicação do processo de DM visto que este, apesar de bem estabelecido, é pouco aplicado para tratamento de águas residuais e posterior reuso de água na indústria têxtil. Além disso, destaca-se a contribuição deste trabalho com corantes disperso, fato este não avaliado na literatura.

\section{NOMENCLATURA}

Área efetiva da membrana: A $\left(\mathrm{m}^{2}\right)$

Concentração de corante na alimentação: $\mathrm{C}\left(\mathrm{mg} \cdot \mathrm{L}^{-1}\right)$

Fluxo permeado: $\mathrm{J}\left(\mathrm{kg} \cdot \mathrm{m}^{-2} \cdot \mathrm{h}^{-1}\right)$

Massa coletada no permeado: $\Delta \mathrm{M}(\mathrm{kg})$

Recuperação de corante: $\mathrm{R}(\%)$

Tempo: $\mathrm{t}(\mathrm{h})$

\section{REFERÊNCIAS}

ALKHUDHIRI, A.; DARWISH, N.; HILAL, N. Membrane distillation: A comprehensive review. Desal., n. 287, p. 2-18, 2011.

DRIOLI, E.; ALI, A.; MACEDONIO, F. Membrane distillation: Recent developments and perspectives. Desal., n. 356, p. 56-84, 2014.

MOKHTAR, N. M.; LAU, W. J.; ISMAIL, A. F; KARTOHARDJONO, S.; LAI, S. O.; TEOH, H. C. The potential of direct contact membrane distillation for industrial textile wastewater treatment using PVDF-Cloisite 15A nanocomposite membrane. Chem. Eng. Res. Des., n. 111, p. 284-293, 2016.

MOZIA, S.; MORAWSKI, A. W.; TOYODA, M.; TSUMURA, T. Integration of photocatalysis and membrane distillation for removal of mono- and poly-azo dyes from water. Desal., n. 250, p. 666-672, 2009.

SERT, G.; BUNANI, S.; KABAY, N.; EGEMEN, Ö.; ARDA, M.; PEK, T. Ö.; YÜKSEL, M. Investigation of mini pilot scale MBR-NF and MBR-RO integrated systems performance - preliminary field tests. J. Wat. Proc. Eng., n. 12, p. 72-77, 2016. 